\title{
Eco-floristic study of weed flora of wheat crop under edaphic variation in tehsil Razar district Swabi, Khyber Pakhtunkhwa, Pakistan
}

\author{
Maqsood Anwar ${ }^{1 *}$, Naveed Akhtar ${ }^{1}$, Shah Khalid ${ }^{1}$ and Sumbul Qadar ${ }^{1}$ \\ 1. Department of Botany, Islamia College Peshawar, Pakistan \\ *Corresponding author's email: maqsoodanwarbot@yahoo.com \\ Citation \\ Maqsood Anwar, Naveed Akhtar, Shah Khalid and Sumbul Qadar. Eco-floristic study of weed flora of wheat crop \\ under edaphic variation in tehsil Razar district Swabi, Khyber Pakhtunkhwa, Pakistan. Pure and Applied Biology. \\ Vol. 11, Issue 1, pp1-10. http://dx.doi.org/10.19045/bspab.2022.110001

\begin{tabular}{llll}
\hline \hline Received: 04/01/2021 & Revised: 26/03/2021 & Accepted: 05/04/2021 & Online First: 17/05/2021 \\
\hline
\end{tabular}

\section{Abstract}

Eco-floristic study of weed flora of wheat crop under edaphic variation was conducted in tehsil Razar district Swabi, Khyber Pakhtunkhwa, Pakistan during February-April 2016. A total of 90 weed species distributed in 72 genera and 26 families were reported from 20 selected wheat fields/sites of the study area. Out of 26 families, 23 were dicots having (58 genera \& 76 species) and 3 were monocots having (14 genera \& 14 species). Fabaceae (14 species), Asteraceae (13 species) and Poaceae (11 species) were dominant families. They were followed by Brassicaceae ( 7 species), Caryophyllaceae (6 species) and Polygonaceae (5 species). The rest of families were represented by 4 or less than 4 species each. Annuals contributed $82(91.1 \%)$ species while perennials shared $8(8.9 \%)$ species. Therophytes and Nanophylls were dominant life form and leaf size contributed 80 (88.9\%) and 42 (46.7\%) species respectively. There were $63(70 \%)$ species with simple leaves, $16(17.8 \%)$ species with compound leaves and 11(12.2\%) species were represented by dissected or decompound leaves. Physico-chemical analysis of soil revealed that soil texture varied from sandy-loam to sandyclay-loam, silt-loam, loam, sandy or loamy-sand. Soil pH ranged from 5.8 to 8.1. Soil Electrical conductivity (EC) varied from $0.06 \mathrm{dsm}^{-1}$ to $0.23 \mathrm{dsm}^{-1}$. Soil Calcium carbonates (CaCO3) ranged from $5.5 \%$ to $23.5 \%$. While soil organic matter $(\mathrm{OM})$ varied from $0.15 \%$ to $1.94 \%$. It was concluded that soil texture, $\mathrm{pH}$, electrical conductivity, organic matter and Calcium carbonates were strong edaphic variables that gives rise to diverse weed species composition.

Keywords: Floristic composition; Life form; Razar; Soil; Weeds; Wheat

\section{Introduction}

Razar is a tehsil of district Swabi Khyber Pakhtunkhwa, Pakistan. It is lies from $34^{\circ}$ $6^{\prime} 0^{\prime \prime}$ to $34^{\circ} 23^{\prime} 0^{\prime \prime}$ North latitudes and $72^{\circ}$ $12^{\prime} 0^{\prime \prime}$ to $72^{\circ} 27^{\prime} 0^{\prime \prime}$ East longitudes. The total area of tehsil Razar is approximately $385 \mathrm{Km}^{2}$ and its population is 583,936 as per census of 2017. Soil of the area is fertile and used for general cropping. It shows great variation in color and texture. Tehsil
Razar has extreme climate. Summer is long and very hot. June, July and August records quite high temperature. Dust storms are very frequent at night during summer. A rapid fall in temperature recorded from October onwards. Winter is very cold. January is coldest month. July and August (monsoon) receive maximum rainfall during which the weather become hot and 
humid. Maximum humidity has been recorded in monsoon and in the month of December [1].

Weeds are those plants which grows so plentifully or luxuriantly that are harmful to crops, human and animals. Weeds are unwanted plants growing in cultivated as well as in domesticated areas and adapted to various edaphic and climatic conditions. Plant species of family Asteraceae, Brassicaceae, Papilionaceae and Poaceae constitutes major weed flora in cultivated agricultural crops. There are almost 30,000 weed plants in worlds, of them, more than 50 causing significant damage to agricultural crops [2]. Weeds are hidden enemies and strong competitors of crops, competes with crops mainly for water, light, nutrients and space and decreases quality as well as quantity of crop [3]. Some weeds secrete certain allelochemicals which affect the germination and growth of agricultural crops [4]. Floristic composition is a characteristic set of plant species within a plant community. Floristic list is valuable because each species has relation to the environment and other species and has its own ecological amplitude and good indicator value in a community. A lot of climatic, edaphic and biological factors influence the floristic composition. The general appearance of the plant body is referred to its life form. Relative proportion or percentage expression of distribution of different life forms and leaf size in the flora of an area is termed as "biological spectrum" and "leaf size spectrum". Life form and leaf size spectra are two physiognomic attributes and indicators of climate of an area that have been generally used in vegetation analysis [5]. The present study aimed Eco-floristic analysis of weeds of wheat crop under edaphic variation in tehsil Razar district Swabi, Khyber Pakhtunkhwa, Pakistan.

\section{Materials and Methods}

Plant collection, drying, mounting and identification

Floristic study of weeds was conducted in wheat fields during February-April, 2016.
Weed plants were thoroughly collected from 20 selected wheat fields. The collected specimens were dried in papers, preserved properly and then mounted on standard herbarium sheets. All these plants were identified with the help of Flora of Pakistan [6-8]. The identification was later on confirmed by using online Flora of Pakistan-Tropicos

(www.tropicos.org/Project/Pakistan) and The Plant List (www.theplantlist.org.). The voucher specimens after identification were numbered and submitted for future references in the herbarium, Department of Botany, Islamia College Peshawar.

\section{Ecological attributes and soil analysis}

Weed plants were then classified into various life form and leaf size classes following $[5,9,10]$. A complete floristic list of weeds with ecological attributes was compiled alphabetically. Soil samples were collected from $0-20 \mathrm{~cm}$ depth at all 20 selected wheat fields. Soil texture was determined by Hydrometer method [11]. Texture triangle of [12] was used for determination of texture classes. Soil $\mathrm{pH}$ was determined with the help of $\mathrm{pH}$ meter $[13,14]$. Electrical conductivity (EC) was determined with the help of electrical conductivity meter [14]. Soil organic matter was determined following method of [15]. Calcium carbonate was determined by acid neutralization method [16].

\section{Results and Discussion Floristic composition}

The weed flora of wheat in study area consisted of 90 species distributed in 72 genera and 26 families. Out of 26 families, 3 were monocots having (14 genera and 14 species) and 23 were dicots having (58 genera and 76 species) (Table 1). Annuals shared $82(91.1 \%)$ species while perennials contributed $8(8.9 \%)$ species (Table 2$)$. Fabaceae (14 species), Asteraceae (13 species) and Poaceae (11 species) were dominant families. They were followed by Brassicaceae (7 species), Caryophyllaceae (6 species) and Polygonaceae (5 species) (Table 1; Fig. 1). The present results are in line with [17] who recorded 83 weed 
species distributed in 26 families from three wheat growing areas of Quetta, Pakistan. [18] recorded 93 weed species distributed in 34 families from FR Bannu, Pakistan. [19] listed 91 weeds from wheat fields of district Bannu, Pakistan. While studying diversity of weeds in Rabi Wheat crops, [20] reported 76 weed species distributed in 27 families. In the present work, the dominant families were Fabaceae, Asteraceae, Poaceae, Brassicaceae, Caryophyllaceae and Polygonaceae. [21] reported Fabaceae, Asteraceae, Poaceae, Brassicaceae and Caryophyllaceae as dominant families from Dera Ismail Khan, Pakistan while working on weeds of wheat. In the study of [22], Fabacae was dominant family in district Khairpur, Sindh, Pakistan.

\section{Life form spectra/Biological spectra}

Life form spectra revealed that therophytes were dominant life form comprised 80 $(88.9 \%)$ species. Hemicryptophytes shared $7(7.8 \%)$ species followed by geophytes 2 (2.2\%) species and chamaephytes $1(1.1 \%)$ species (Table 2; Fig. 2). Our results are in line with the studies of other workers [17, 23-25] who recorded therophytes as dominant group followed by hemicryptophytes, chamaephytes and geophytes. In the study of [26, 27] therophytes were dominant life form which support the present findings. Similarly, in the study of $[28,29]$ therophytes were dominant life form followed by geophytes.

\section{Leaf size spectra}

Leaf size spectra indicated that nanophylls were dominant group (42 spp; 46.7\%) followed by microphylls (32 spp; 35.6\%), leptophylls (13 spp; 14.4\%) and mesophylls (3 spp; 3.3\%) (Table 2; Fig. 3). The present results support the finding of $[26,27]$ who recorded nanophylls as major group. The present findings are in contrast with [24, 29] who recorded microphylls as a major leaf size class followed by nanophylls and leptophylls.

\section{Lamina shape/Leaf types}

Leaves are the most important organs of most vascular plants. There are so many types of leaves in nature. Flowering plants have either simple, compound or dissected leaves. There were $63(70 \%)$ species with simple leaves, 16 (17.8\%) species with compound leaves and 11(12.2\%) species were represented by dissected or decompound leaves (Table 2).

\section{Soil analysis}

Physico-chemical analysis of soil revealed that texture varied from sandy-loam to sandy-clay-loam, silt-loam, loam, sandy or loamy-sand. Soil pH ranged from 5.8 to 8.1. Electrical conductivity (EC) ranged from $0.06 \mathrm{dsm}-1$ to $0.23 \mathrm{dsm}-1$. Calcium carbonates $(\mathrm{CaCO} 3)$ varied from $5.5 \%$ to $23.5 \%$. While organic matter $(\mathrm{OM})$ varied from $0.15 \%$ to $1.94 \%$ (Table 3 ). Soil is main abiotic ecological factor that play important role in the distribution of plant species composition in particular area. In the present study soil texture, $\mathrm{pH}$, electrical conductivity, organic matter and Calcium carbonates were strong edaphic variables that gives rise to diverse weed species composition. Soil texture, $\mathrm{pH}$, electrical conductivity, organic matter and carbonates play key role in weed species diversity. [30] stated that texture, $\mathrm{pH}$, electrical conductivity, organic matter and carbonates play an important role in weed species diversity. [31] described that soil organic matter is main soil fertility factor that affects the distribution of plant diversity. Furthermore, [32] reported that soil texture control infiltration rate, water holding capacity, availability of moisture and plant nutrition. Soil texture, organic carbon and salinity are main three factors for weed species composition [33-35]. Generally, the soil of the research area is basic and rarely acidic. Compare to [36] who found same type of soil $\mathrm{pH}$ ranges 6.5-7.5 from Doon Valley India while studying weeds of wheat crop, which is according to our findings. [37] analyzed three soil series for texture, $\mathrm{pH}$, electrical conductivity and organic matter. Similarly, [38] conducted phytosociological study of weeds of wheat crop under edaphic variations i.e. soil texture, $\mathrm{pH}$, electrical conductivity and organic matter. 
Table 1. Floristic list with ecological characteristics of weed flora of wheat crop in tehsil Razar district Swabi, Pakistan

\begin{tabular}{|c|c|c|c|c|c|}
\hline S. No. & Divisions/Families/Species & $\mathbf{L F}$ & $\mathbf{L S}$ & Life span & Lamina \\
\hline \multicolumn{6}{|c|}{ A. MONOCOTYLEDONAE } \\
\hline \multicolumn{6}{|c|}{ 1. Cyperaceae } \\
\hline 1 & Cyperus rotundus $\mathrm{L}$. & $\mathrm{Ge}$ & Nan & Perennial & Simple \\
\hline 2 & Schoenoplectus triqueter (L.) Palla & $\mathrm{Ge}$ & Nan & Perennial & Simple \\
\hline \multicolumn{6}{|c|}{ 2. Juncaceae } \\
\hline 3 & Juncus bufonius L. & Th & Lep & Annual & Simple \\
\hline \multicolumn{6}{|c|}{ 3. Poaceae } \\
\hline 4 & Alopecurus myosuroides Huds. & Th & Mic & Annual & Simple \\
\hline 5 & Avena sativa $\mathrm{L}$. & $\mathrm{Th}$ & Mic & Annual & Simple \\
\hline 6 & Bromus pectinatus Thunb. & Th & Nan & Annual & Simple \\
\hline 7 & Cynodon dactylon (L.) Pers. & $\mathrm{Hc}$ & Lep & Perennial & Simple \\
\hline 8 & Desmostachya bipinnata (L.) Stapf & $\mathrm{Hc}$ & Mic & Perennial & Simple \\
\hline 9 & Phalaris minor Retz. & $\mathrm{Th}$ & Mic & Annual & Simple \\
\hline 10 & Phleum paniculatum Huds. & Th & Mic & Annual & Simple \\
\hline 11 & Poa annua $\mathrm{L}$. & Th & Lep & Annual & Simple \\
\hline 12 & Polypogon monspeliensis (L.) Desf. & $\mathrm{Th}$ & Nan & Annual & Simple \\
\hline 13 & Rostraria cristata $(\mathrm{L}$.$) Tzvelev$ & Th & Nan & Annual & Simple \\
\hline 14 & Saccharum ravennae (L.) L. & $\mathrm{Hc}$ & Nan & Perennial & Simple \\
\hline \multicolumn{6}{|c|}{ B. DICOTYLEDONAE } \\
\hline \multicolumn{6}{|c|}{ 4. Amaranthaceae } \\
\hline 15 & Amaranthus viridis L. & Th & Mic & Annual & Simple \\
\hline 16 & Chenopodium album $\mathrm{L}$. & Th & Mic & Annual & Simple \\
\hline 17 & C. murale $\mathrm{L}$. & Th & Mic & Annual & Simple \\
\hline 18 & C. strictum Roth. & Th & Nan & Annual & Simple \\
\hline \multicolumn{6}{|c|}{ 5. Apiaceae } \\
\hline 19 & Scandix pecten-veneris $\mathrm{L}$. & Th & Mic & Annual & Dissected \\
\hline 20 & Torilis leptophylla (L.) Rchb.f. & Th & Mic & Annual & Dissected \\
\hline \multicolumn{6}{|c|}{ 6. Asteraceae } \\
\hline 21 & Calendula arvensis $\mathrm{L}$. & Th & Nan & Annual & Simple \\
\hline 22 & Carthamus lanatus L. & Th & Mic & Annual & Dissected \\
\hline 23 & C. oxyacantha M.Bieb. & $\mathrm{Th}$ & Mic & Annual & Simple \\
\hline 24 & Centaurea iberica Trevir. ex Spreng. & Th & Mic & Annual & Dissected \\
\hline 25 & Cirsium arvense (L.) Scop. & Th & Mic & Annual & Simple \\
\hline 26 & Conyza canadensis (L.) Cronquist & $\mathrm{Th}$ & Mic & Annual & Simple \\
\hline 27 & C. sumatrensis (S.F.Blake) Pruski \& G.Sancho & Th & Mic & Annual & Simple \\
\hline 28 & Gamochaeta pensylvanica (Willd.) Cabrera & Th & Nan & Annual & Simple \\
\hline 29 & Parthenium hysterophorus L. & Th & Mes & Annual & Dissected \\
\hline 30 & Silybum marianum (L.) Gaertn. & Th & Mes & Annual & Simple \\
\hline 31 & Sonchus oleraceus $(\mathrm{L}.) \mathrm{L}$. & Th & Mic & Annual & Dissected \\
\hline 32 & Taraxacum officinale (L.) Weber ex F.H.Wigg. & $\mathrm{Hc}$ & Mic & Annual & Dissected \\
\hline 33 & Xanthium strumarium $\mathrm{L}$. & Th & Mes & Annual & Simple \\
\hline
\end{tabular}




\begin{tabular}{|c|c|c|c|c|c|}
\hline 34 & Buglossoides arvensis (L.) I.M.Johnst. & Th & Nan & Annual & Simple \\
\hline 35 & $\begin{array}{l}\text { Bothriospermum tenellum (Hornem.) Fisch. \& } \\
\text { C.A.Mey. }\end{array}$ & Th & Nan & Annual & Simple \\
\hline \multicolumn{6}{|c|}{ 8. Brassicaceae } \\
\hline 36 & Brassica campestris L. & Th & Mic & Annual & Dissected \\
\hline 37 & Capsella bursa-pastoris (L.) Medik. & Th & Mic & Annual & Simple \\
\hline 38 & Cardaria chalepensis (L.) Hand-Mazz. & Th & Mic & Annual & Simple \\
\hline 39 & Coronopus didymus (L.) Sm. & $\mathrm{Hc}$ & Nan & Annual & Dissected \\
\hline 40 & Malcolmia cabulica (Boiss.) Hook. f. \& Thomson & Th & Nan & Annual & Simple \\
\hline 41 & Nasturtium officinale W.T. Aiton & Th & Nan & Annual & Simple \\
\hline 42 & Neslia apiculata Fisch., C.A.Mey. \& Avé-Lall. & Th & Mic & Annual & Simple \\
\hline \multicolumn{6}{|c|}{ 9. Cannabaceae } \\
\hline 43 & Cannabis sativa $\mathrm{L}$. & Th & Nan & Annual & Compound \\
\hline \multicolumn{6}{|c|}{ 10. Caryophyllaceae } \\
\hline 44 & Arenaria serpyllifolia Bourg. ex Willk. \& Lange & Th & Lep & Annual & Simple \\
\hline 45 & Cerastium dichotomum $\mathrm{L}$. & Th & Nan & Annual & Simple \\
\hline 46 & C. glomeratum Thuill. & Th & Nan & Annual & Simple \\
\hline 47 & Silene conoidea $\mathrm{L}$. & Th & Mic & Annual & Simple \\
\hline 48 & Spergula arvensis $\mathrm{L}$. & Th & Lep & Annual & Simple \\
\hline 49 & Stellaria media (L.) Vill. & Th & Nan & Annual & Simple \\
\hline \multicolumn{6}{|c|}{ 11. Convolvulaceae } \\
\hline 50 & Convolvulus arvensis $\mathrm{L}$. & Th & Nan & Annual & Simple \\
\hline \multicolumn{6}{|c|}{ 12. Euphorbiaceae } \\
\hline 51 & Euphorbia esula L. & Th & Lep & Annual & Simple \\
\hline 52 & E. helioscopia L. & Th & Nan & Annual & Simple \\
\hline 53 & E. prostrata Aiton & Th & Lep & Annual & Simple \\
\hline \multicolumn{6}{|c|}{ 13. Fabaceae } \\
\hline 54 & Astragalus hamosus Bal. ex Bunge & Th & Nan & Annual & Compound \\
\hline 55 & Lathyrus aphaca $\mathrm{L}$. & Th & Nan & Annual & Compound \\
\hline 56 & $\begin{array}{l}\text { Medicago laciniata (L.) Mill. Var. brachycantha } \\
\text { Boiss. }\end{array}$ & Th & Nan & Annual & Compound \\
\hline 57 & M. lupulina $\mathrm{L}$. & Th & Nan & Annual & Compound \\
\hline 58 & M. minima (L.) L. & Th & Lep & Annual & Compound \\
\hline 59 & M. polymorpha $\mathrm{L}$. & Th & Nan & Annual & Compound \\
\hline 60 & Melilotus indica (L.) All. & Th & Nan & Annual & Compound \\
\hline 61 & Trifolium resupinatum $\mathrm{L}$. & Th & Nan & Annual & Compound \\
\hline 62 & Trigonella monantha C.A. Mey. & Th & Nan & Annual & Compound \\
\hline 63 & Vicia bithynica (L.) L. & Th & Nan & Annual & Compound \\
\hline 64 & V. hirsuta (L.) Gray & Th & Lep & Annual & Compound \\
\hline 65 & V. peregrina $\mathrm{L}$. & Th & Nan & Annual & Compound \\
\hline 66 & V. sativa $\mathrm{L}$. & Th & Nan & Annual & Compound \\
\hline 67 & V. tetrasperma (L.) Schreb. & Th & Lep & Annual & Compound \\
\hline \multicolumn{6}{|c|}{ 14. Fumariaceae } \\
\hline 68 & Fumaria indica Pugsley & Th & Lep & Annual & Dissected \\
\hline
\end{tabular}


Anwar et al.

\begin{tabular}{|c|c|c|c|c|c|}
\hline 69 & Centaurium pulchellum (Sw.) Druce & $\mathrm{Th}$ & Nan & Annual & Simple \\
\hline \multicolumn{6}{|c|}{ 16. Illecebraceae } \\
\hline 70 & Herniaria hirsuta $\mathrm{L}$. & $\mathrm{Th}$ & Lep & Annual & Simple \\
\hline \multicolumn{6}{|c|}{ 17. Lamiaceae } \\
\hline 71 & Mentha longifolia $(\mathrm{L}.) \mathrm{L}$. & $\mathrm{Hc}$ & Mic & Perennial & Simple \\
\hline 72 & Salvia plebeia $\mathrm{R} . \mathrm{Br}$. & Th & Mic & Annual & Simple \\
\hline \multicolumn{6}{|c|}{ 18. Malvaceae } \\
\hline 73 & Malva neglecta Wallr. & $\mathrm{Th}$ & Mic & Annual & Simple \\
\hline \multicolumn{6}{|c|}{ 19. Oxalidaceae } \\
\hline 74 & Oxalis corniculata $\mathrm{L}$. & $\mathrm{Th}$ & Nan & Annual & Compound \\
\hline \multicolumn{6}{|c|}{ 20. Papaveraceae } \\
\hline 75 & Papaver hybridum L. & Th & Nan & Annual & Dissected \\
\hline \multicolumn{6}{|c|}{ 21. Polygonaceae } \\
\hline 76 & Emex spinosa (L.) Campd. & $\mathrm{Th}$ & Nan & Annual & Simple \\
\hline 77 & Persicaria barbata (L.) H. Hara & $\mathrm{Th}$ & Mic & Annual & Simple \\
\hline 78 & Polygonum plebeium R.Br. & Th & Nan & Annual & Simple \\
\hline 79 & Rumex chalepensis Mill. & $\mathrm{Ch}$ & Mic & Perennial & Simple \\
\hline 80 & R. dentatus $\mathrm{L}$. & Th & Mic & Annual & Simple \\
\hline \multicolumn{6}{|c|}{ 22. Primulaceae } \\
\hline 81 & Anagallis arvensis $\mathrm{L}$. & Th & Nan & Annual & Simple \\
\hline \multicolumn{6}{|c|}{ 23. Ranunculaceae } \\
\hline 82 & Ranunculus arvensis L. & $\mathrm{Th}$ & Mic & Annual & Simple \\
\hline 83 & R. cantoniensis DC. & $\mathrm{Th}$ & Mic & Annual & Simple \\
\hline 84 & R. muricatus L. & Th & Mic & Annual & Simple \\
\hline \multicolumn{6}{|c|}{ 24. Rubiaceae } \\
\hline 85 & Galium aparine L. & $\mathrm{Th}$ & Lep & Annual & Simple \\
\hline \multicolumn{6}{|c|}{ 25. Scrophulariaceae } \\
\hline 86 & Mazus pumilus (Burm.f.) Steenis & $\mathrm{Th}$ & Nan & Annual & Simple \\
\hline 87 & Misopates orontium (L.) Raf. & Th & Nan & Annual & Simple \\
\hline 88 & Veronica anagallis-aquatica $\mathrm{L}$. & Th & Nan & Annual & Simple \\
\hline 89 & V. persica Poir. & Th & Nan & Annual & Simple \\
\hline \multicolumn{6}{|c|}{ 26. Verbenaceae } \\
\hline 90 & Phyla nodiflora (L.) Greene & $\mathrm{Hc}$ & Nan & Perennial & Simple \\
\hline
\end{tabular}

Key: LF: Life form, LS: Leaf Size, Th: Therophytes, Hc: Hemicryptophytes, Ge: Geophytes, Ch: Chamaephytes, Lep: Leptophylls, Mic: Microphylls, Mes: Mesophylls, Nan: Nanophylls 


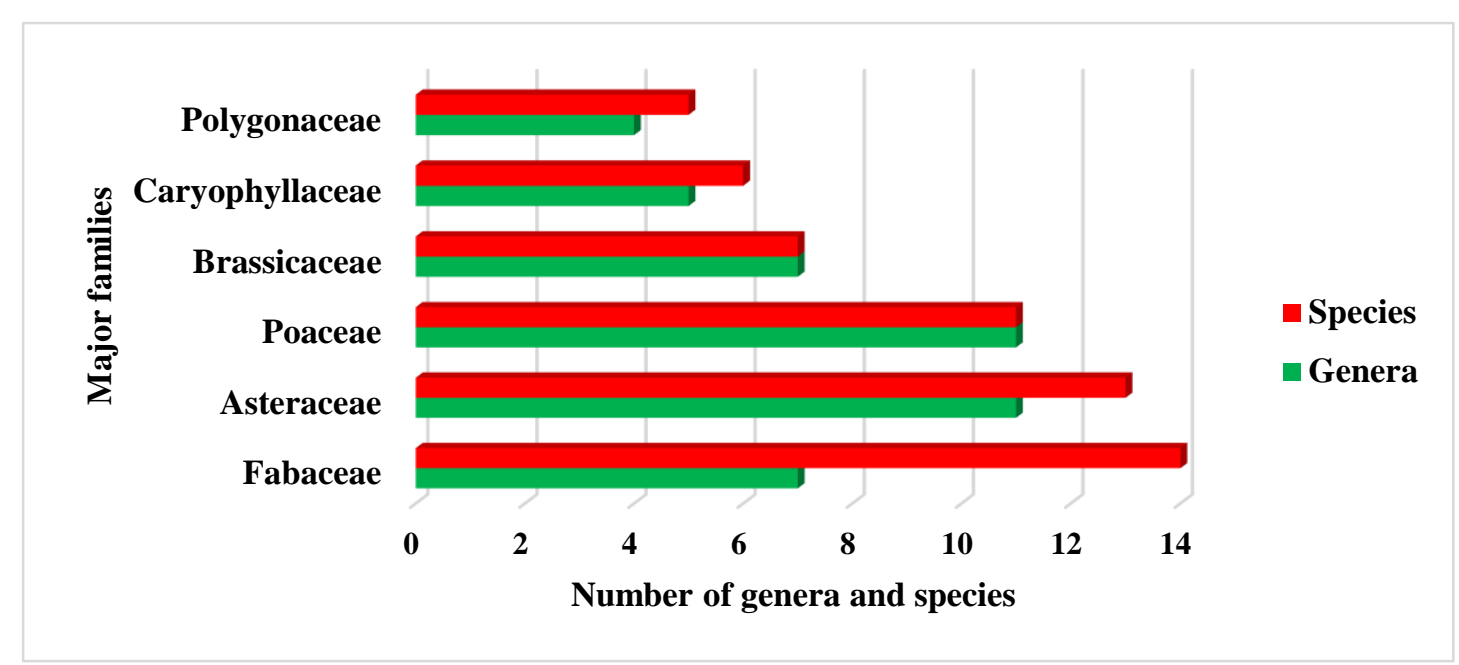

Figure 1. Distribution of genera and species in major families.

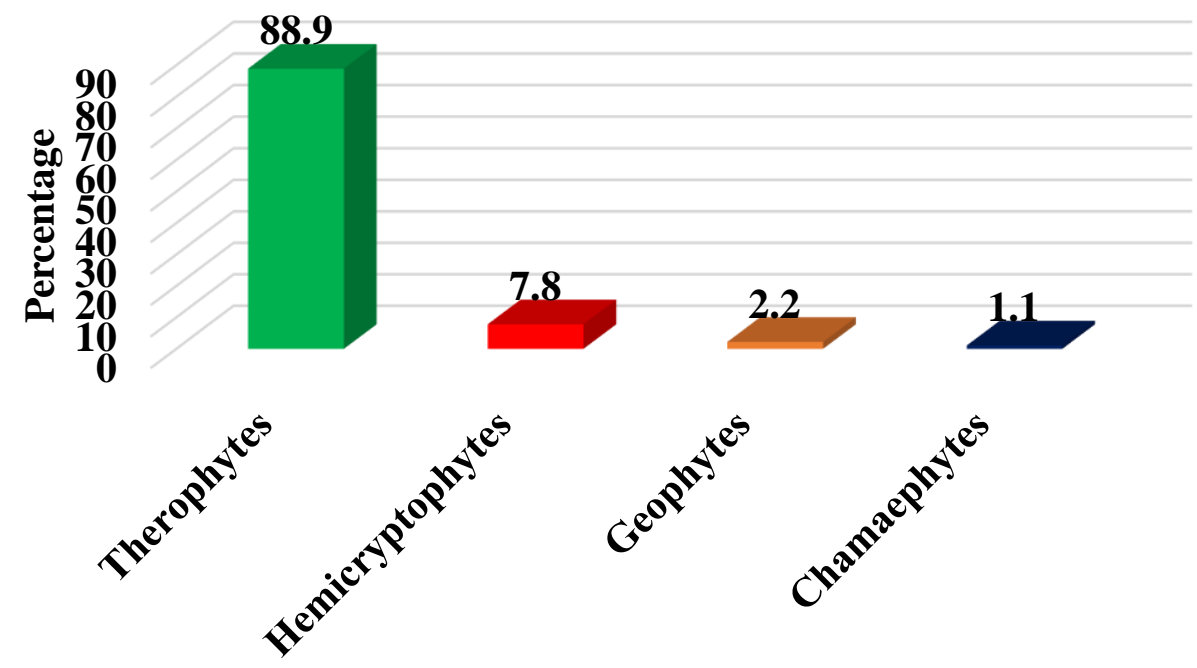

Life form

Figure 2. Life form spectra of weed species of wheat crop.

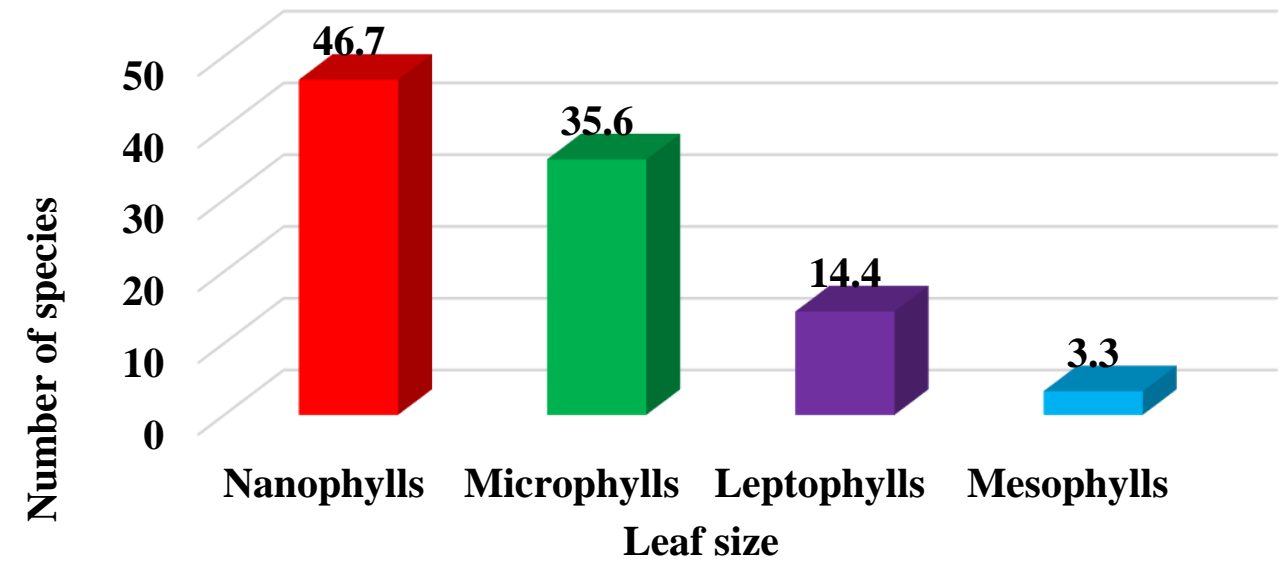

Figure 3. Life size spectra of weed species of wheat crop. 
Table 2. Summary of floristic composition, life form spectra, leaf size spectra and leaf types of weeds of wheat crop in tehsil Razar district Swabi, Pakistan

\begin{tabular}{|c|c|c|c|}
\hline S. No. & Ecological characteristics & Number of Species & Percentage \\
\hline \multicolumn{4}{|c|}{ Weed flora } \\
\hline I & Total species & 90 & --- \\
\hline II & Total genera & 72 & --- \\
\hline III & Total families & 26 & --- \\
\hline \multicolumn{4}{|c|}{ Life span } \\
\hline I & Annuals & 82 & 91.1 \\
\hline II & Perennials & 08 & 8.9 \\
\hline \multicolumn{4}{|c|}{ Life form spectra } \\
\hline I & Therophytes & 80 & 88.9 \\
\hline II & Hemicryptophytes & 07 & 7.8 \\
\hline III & Chamaephytes & 02 & 2.2 \\
\hline IV & Geophytes & 01 & 1.1 \\
\hline \multicolumn{4}{|c|}{ Leaf size spectra } \\
\hline I & Nanophylls & 42 & 46.7 \\
\hline II & Microphylls & 32 & 35.6 \\
\hline III & Leptophylls & 13 & 14.4 \\
\hline IV & Mesophylls & 03 & 3.3 \\
\hline \multicolumn{4}{|c|}{ Lamina shape/Leaf types } \\
\hline I & Simple & 63 & 70.0 \\
\hline II & Compound & 16 & 17.8 \\
\hline III & Dissected/Decompound & 11 & 12.2 \\
\hline
\end{tabular}

Table 3. Physico-chemical characteristics of soil of different wheat fields/sites in tehsil Razar, district Swabi, Pakistan

\begin{tabular}{|c|c|c|c|c|c|c|c|c|}
\hline \multirow{2}{*}{ Sites } & \multirow{2}{*}{ pH (1:5) } & EC (1:5) & $\mathrm{CaCO}_{3}$ & O.M & Clay & Silt & Sand & \multirow{2}{*}{ Textural class } \\
\hline & & $\mathrm{dsm}^{-1}$ & \multicolumn{2}{|c|}{$\%$} & \multicolumn{3}{|c|}{$\%$} & \\
\hline Site-1 & 7.8 & 0.13 & 18.5 & 0.77 & 15.2 & 22.6 & 62.2 & Sandy loam \\
\hline Site-2 & 7.7 & 0.12 & 19.5 & 1.03 & 19.2 & 28.6 & 52.2 & Sandy loam \\
\hline Site-3 & 7.4 & 0.15 & 17.2 & 0.25 & 3.2 & 18.8 & 78.0 & Loamy sand \\
\hline Site-4 & 7.3 & 0.16 & 16.6 & 1.13 & 9.2 & 1.4 & 89.4 & Sandy \\
\hline Site-5 & 5.8 & 0.14 & 12.5 & 1.81 & 23.2 & 27.2 & 49.6 & Sandy clay loam \\
\hline Site-6 & 7.6 & 0.20 & 16.8 & 1.03 & 17.2 & 8.8 & 74.0 & Sandy loam \\
\hline Site-7 & 7.8 & 0.11 & 22.2 & 1.29 & 17.2 & 14.0 & 68.8 & Sandy loam \\
\hline Site-8 & 8.1 & 0.08 & 18.7 & 1.03 & 13.4 & 4.2 & 82.4 & Loamy sand \\
\hline Site-9 & 6.9 & 0.13 & 5.5 & 0.51 & 11.8 & 54.8 & 33.4 & Silt loam \\
\hline Site-10 & 7.4 & 0.06 & 11.0 & 0.51 & 13.2 & 5.4 & 81.4 & Sandy loam \\
\hline Site-11 & 7.4 & 0.15 & 6.5 & 0.77 & 17.0 & 29.8 & 53.2 & Sandy loam \\
\hline Site-12 & 6.0 & 0.14 & 10.7 & 1.38 & 20.6 & 26.0 & 53.4 & Sandy clay loam \\
\hline Site-13 & 7.2 & 0.16 & 23.5 & 0.25 & 8.6 & 49.8 & 41.6 & Loam \\
\hline Site-14 & 7.3 & 0.14 & 10.5 & 1.03 & 17.2 & 4.6 & 78.2 & Sandy loam \\
\hline Site-15 & 6.5 & 0.12 & 10.5 & 1.24 & 16.0 & 8.6 & 75.4 & Sandy loam \\
\hline Site-16 & 6.8 & 0.10 & 6.5 & 1.94 & 12.2 & 4.4 & 83.4 & Loamy sand \\
\hline Site-17 & 7.8 & 0.10 & 14.2 & 0.39 & 3.8 & 48.6 & 47.6 & Sandy loam \\
\hline Site-18 & 7.6 & 0.15 & 13.0 & 1.60 & 9.2 & 2.0 & 88.8 & Loamy sand \\
\hline Site-19 & 7.6 & 0.23 & 14.6 & 1.03 & 23.2 & 6.8 & 70.0 & Sandy clay loam \\
\hline Site-20 & 7.9 & 0.17 & 16.0 & 0.15 & 4.6 & 52.0 & 43.4 & Silt loam \\
\hline
\end{tabular}




\section{Conclusion}

The present findings suggest that a large number of weed plants infesting wheat crop in the study area may causes loss to crop yields. For acquiring better yield, it is important to take appropriate biological, chemical and mechanical measurement for weed control.

\section{Authors' contributions}

Conceived and designed the experiments: N Akhtar, Performed the experiments: M Anwar, Analyzed the data: M Anwar, Contributed reagents/ materials/ analysis tools: S Khalid \& S Qadar, Wrote the paper: M Anwar.

\section{References}

1. Anwar M, Khan WM, Khan MS, Murad W \& Ali S (2015). Taxonomic study of Family Papilionaceae of District Swabi, Khyber Pakhtunkhwa, Pakistan. Pure Appl Biol 4(1):125-128.

2. Mahmood TZ \& Niaz SA (1992). "Weeds in Cropped Land at Islamabad," NARC, Identification and control of weeds manual, national training course, Pakistan Agriculture Research Council, Islamabad. pp.79.

3. Dangwal LR, Singh A, Singh T, Sharma A \& Sharma C (2010). Common weeds of Rabi (winter) crops of Tehsil Nowshera, District Rajouri (Jammu \& Kashmir), India. Pak J Weed Sci Res 16(1): 39-45

4. Oudhia P \& Tripathi RS (1998). Allelopathic effects of Parthenium hysterophorus L. on kodo, mustard and problematic weeds. $1^{\text {st }}$ Int. Conf. Parthenium management UAS, Dhaward, India. 6-8 Oct., 1997: 136139.

5. Cain SA \& Castro GM (1959). Manual of Vegetation Analysis. Harper \& Brothers, New York, USA.

6. Nasir E \& Ali SI (Eds.) (1970-1989). Flora of Pakistan (Fascicles series 1190). Islamabad, Karachi.

7. Nasir E \& Ali SI (Eds.) (1972). Flora of West Pakistan: An annotated catalogue of the vascular plants of West Pakistan and Kashmir. Fakhri printing Press Karachi.

8. Ali SI \& Nasir YJ (Eds.) (1989-1991). Flora of Pakistan (Fascicle series). Islamabad, Karachi.

9. Raunkiaer C (1934). The life form of plants and statistical plant geography. The Clarendon Press, Oxford.

10. Hussain F (1989). Field and Laboratory Manual for Plant Ecology. University Grants Commission, Islamabad.

11. Bouyoucos GJ (1936). Directions for making mechanical analysis of soils by the hydrometer method. Soil Sci 42: 225-228.

12. Brady NC (1990). The Nature and Properties of Soils. 10th Ed. Macmillan Publishing Co. New York. pp. 621

13. Jackson ML (1962). Soil chemical Analysis. Constable \& Co., Ltd., London. pp. 406-407.

14. Rhoades JD, Miyamoto S \& Westerman RL (1990). Testing soils for salinity and sodicity. Soil Test. Plant Anal 299-336.

15. Black CA (1965). Methods of Soil Analysis. Agron Inc. Madison Wisconsin, USA.

16. Richard LA (1954). Diagnoses and improvement of saline and alkali soils. Agriculture Hand Book, 60: USDA, USA.

17. Hussain F, Rehman SU \& Dasti AA (1985). Studies on weeds of wheat in Quetta. Pak J Agri Res 6(1): 1-7.

18. Ihsanullah, Wazir SM, Farooq A, Khan SU \& Hussain Z (2011). Identification of common weeds and its distribution pattern in wheat fields of FR Bannu, Khyber Pakhtunkhwa, Pakistan. Pak J Weed Sci Res 17(4): 407-416.

19. Khan RU, Mehmood S, Khan SU, Muhammad A \& Hussain Z (2013). Camparative study of weed species recorded in different field crops of Bannu, Khyber Pakhtunkhwa, Pakistan. Pak J Weed Sci Res 20(4): 489-504.

20. Khobragade DP \& Sathawane KN (2014). Weed diversity in Rabi wheat crop of Bhandara District (MS), India. Int J Life Sci Special Issue. 2: 128-131. 
21. Marwat SK, Usman K, Khan NU, Khan MU, Khan EA, Khan MA \& Rehman AU (2013). Weeds of Wheat Crop and Their Control Strategies in Dera Ismail Khan District, Khyber Pakhtunkhwa, Pakistan. Amer J Plant Sci 4: 66-76.

22. Memon RA, Bhatti GR \& Khalid S (2003). Weed diversity of wheat crop in Khairpur district, Sindh. Pak J Weed Sci Res 9(1-2): 99-103.

23. Malik ZH, Awan AA \& Murtaza G (1992). Weeds of wheat fields around Muzaffarabad, Azad Jammu and Kashmir. Pak J Agric Sci 29(1): 61-64.

24. Sher Z, Hussain F, Badshah L \& Wahab M (2011). Floristic composition, communities and ecological characteristics of weeds of wheat fields of Lahor, District Swabi, Pakistan. Pak $J$ Bot 43(6): 2817-2820.

25. Inayat N, Asadullah \& Rashid A (2014). Floristic composition and ecological prevalence of the weed species growing in wheat and sugarcane fields of district Charsadda, Khyber Pakhtunkhwa, Pakistan. Pak J Weed Sci Res 20(3): 405-415.

26. Nasir ZA \& Sultan S (2002). Floristic, biological and leaf size spectra of weeds in Gram, Lentil, Mustard and Wheat fields of district Chakwal, Pakistan. Pak J Biol Sci 5(7): 758-762.

27. Hussain F, Shah SM, Hadi F \& Asadullah (2009). Diversity and Ecological characteristics of weeds of wheat fields of University of Peshawar Botanical Garden at Azakhel, district Nowshera, Pakistan. Pak J Weed Sci Res 15(4): 283-294.

28. Shah SM, Asadullah \& Hadi F (2014). Ecological characteristics of weed flora in the wheat crop of Mastuj valley, district Chitral, Khyber Pakhtunkhwa, Pakistan. Pak J Weed Sci Res 20(4): 479-487.

29. Hadi F \& Ibrar M (2015). Ecology of weeds in wheat crops of Kalash valley, district Chitral, Hindukush range,
Pakistan. Pak J Weed Sci Res 21(3): 425-43

30. Gomaa NH (2012). Composition and diversity of weed communities in $\mathrm{Al}$ Jouf Province, Northern Saudi Arabia. Saudi J Biol Sci 19: 369-376.

31. Zhang K, Dang H, Tan SH, Wang ZH \& Zhang Q (2010). Vegetation community and soil characteristics of abandoned agricultural land and pine plantation in the Qinling Mountains, China. Forest Ecol Manage 259: 20362047.

32. Sperry JS \& Hacke UG (2002). Desert shrub water relations with respect to soil characteristics and plant functional type. Funct Ecol 16: 367-378.

33. Fried G, Norton LR \& Reboud $X$ (2008). Environmental and management factors determining weed species composition and diversity in France. Agric Ecosyst Environ 128: 6876.

34. Andreasen C \& Skovgaard IM (2009). Crop and soil factors of importance for the distribution of plant species on arable fields in Denmark. Agr Ecosyst Environ 133: 61-67.

35. Pinke G, Pal R \& Botta-Dukat $Z$ (2010). Effects of environmental factors on weed species composition of cereal and stubble fields in western Hungary. Cent Eur J Biol 5(2): 283292.

36. Gupta A, Joshi SP \& Manhas RK (2008). Multivariate analysis of diversity and composition of weed communities of wheat fields in Doon Valley, India. Trop Eco 49(2): 103-112.

37. Jakhar GS, Mahar AQ, Abro SA \& Qureshi R (2005). Weed communities of wheat crop under diverse Edaphography of District Khairpur. Pak J Bot 37(3): 709-714.

38. Malik ZH, Amjad MS, Rafique S \& Malik NZ (2013). Phytosociology of some weeds of wheat communities around Kotli fields, Western Himalaya. J Eco Nat Envi 5(11): 340-34. 